\title{
Strangeness Production at CLAS
}

K.H. Hicks for the CLAS Collaboration

Ohio University, Athens, OH, 45701, USA

\begin{abstract}
The CLAS detector is a large acceptance spectrometer located at the electron accelerator at Thomas Jefferson National Accelerator Facility. Production of $K+$ and $K^{*}$ mesons along with associated hyperons were measured using the CLAS detector at electron beam energies of 2.4-4.4 GeV. In addition, polarization transfer from the electron helicity to the A hyperon was measured. These data are compared with theoretical models, where available, based on fits to older, less precise $K+$ data. The new data for CLAS indicate that improvements in the models are necessary in order to interpret the results.
\end{abstract}

\section{INTRODUCTION}

Kaon electroproduction is complementary to pion electroproduction, and comparison of both reactions will be helpful to understand the role of various $N^{*}$ resonances. Since s-quarks are not present as valence quarks within the nucleon, the reaction amplitudes depend on the wavefunction of $s \bar{s}$ quark pairs produced from the vacuum. Most models assume the $s \bar{s}$ are in a ${ }^{3} \mathrm{P}_{0}$ state. Polarization observables, which are easily measured because the A is self-analyzing, provide information about the state of the produced $s \bar{s}$ pair. This in turn can be used in theoretical models to improve our understanding of the reaction mechanism. We may also find that $\mathrm{N}^{*}$ resonances have different decay amplitudes into strange and non-strange channels. Unlike pion production, data for kaon production has been sparse, and the potential for finding "new" resonances is appealing.

In $K+$ production, both t-channel and s-channel diagrams contribute. Because of the heavy mass of the s-quark, the t-channel contributes mostly at forward kaon angles. The $N^{*}$ resonances contribute through the s-channel, and hence will be more visible at backward kaon angles. In addition, only $N^{*}$ resonances can contribute to the $\Lambda$ final state, whereas both $N^{*}$ and $\Delta^{*}$ resonances contribute to the $\Sigma^{0}$ production. Because $\Lambda$ and $\Sigma^{0}$ hyperons have the same valence quarks $(u d s)$ and nearly the same mass, they act as an isospin filter for s-channel production. A
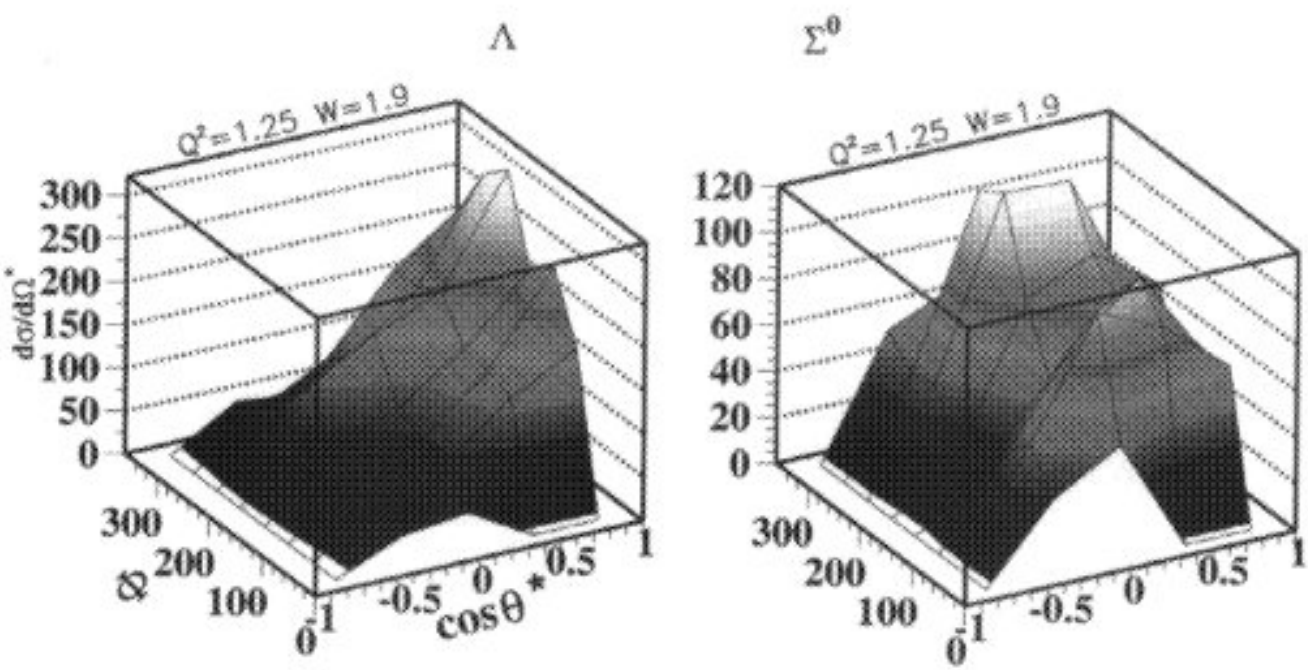

FIGURE 1. Overall angular distribution of cross section to the $\Lambda$ and $\Sigma^{0}$ final states. 
comparison of the W-dependence for these two final states helps to untangle the role of $N^{*}$ and $\Delta^{*}$ resonances in the reaction mechanism.

Other reactions with strange quarks, such as electroproduction of vector mesons like the $K^{*}$ are also useful to understand the role of $N^{*}$ resonances in the reaction mechanism. However, the higher threshold for $K^{*}$ production (above $W=2.0 \mathrm{GeV}$ ) restricts this channel to resonances above the cluster of $L-1$ resonances at about 1.7-1.9 GeV. This may be an advantage in a search for higher lying resonances which may be obscured by t-channel processes in other reaction channels [1].

\section{EXPERIMENTAL SETUP}

The CLAS detector is a novel design with a torroidal magnetic field. The nearly $4 \mathrm{ft}$ coverage is segmented into six sectors, each with three sets of drift chambers followed by plastic scintillators for time-of-flight measurement. At forward angles are Cerenkov detectors and electromagnetic calorimeters used in part for detection of the scattered electron. The CLAS detector has been described in detail elsewhere [2].

\section{RESULTS AND DISCUSSION}

Fig. 1 shows the angular distributions for kaon electroproduction to $\Lambda$ and $\Sigma$ final states. The data for the $\Lambda$ exhibit a large increase as $\theta^{*}$, the angle of the kaon relative to the virtual photon direction, becomes small ( $\cos \theta \sim 1$ ) whereas for the $\Sigma$ data tend to peak at central angles (near $\cos \theta^{*}=0$ ). In addition, the $\varphi$-dependence of the cross sections is very different for these two
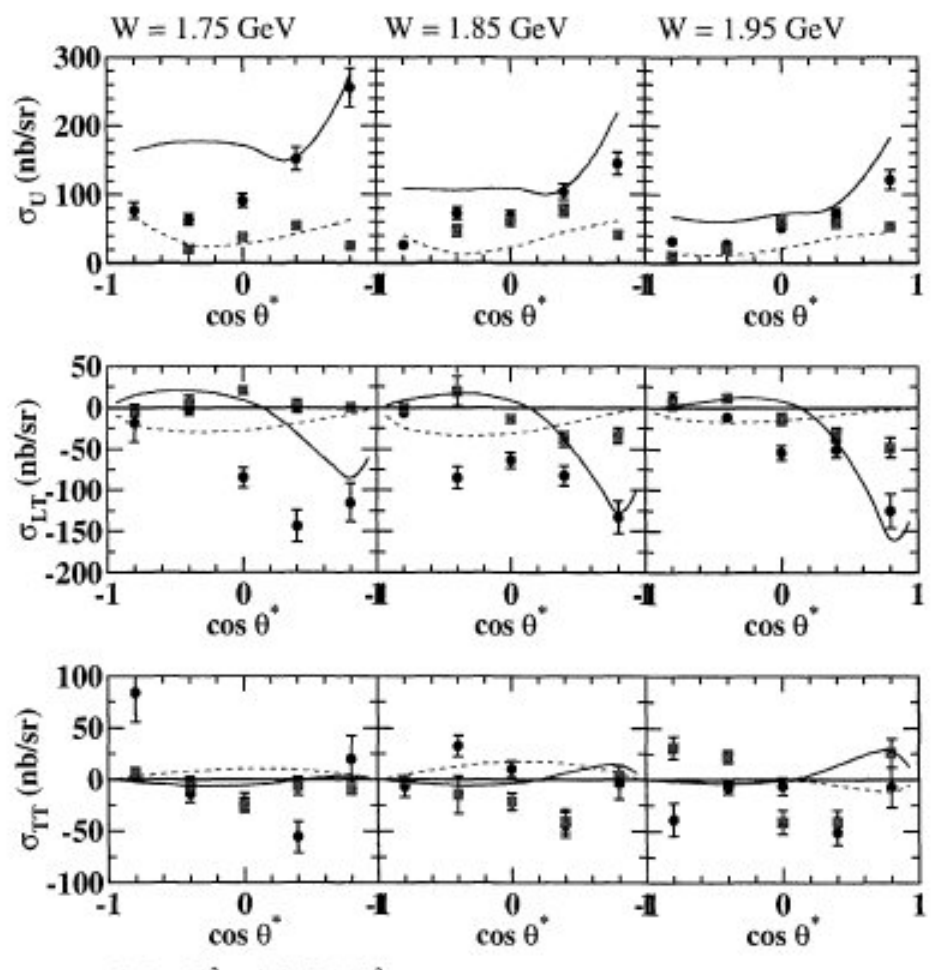

$0.5<\mathrm{Q}^{2}<1.25 \mathrm{GeV}^{2}$

FIGURE 2. Angular distributions of cross sections for different bins in $W$. The $\Lambda$ results are given by circles and the $\Sigma$ results by squares. The curves are calculations for $\Lambda$ (solid) and $\Sigma$ (dashed). 
final states at a given kaon angle. This difference is perhaps surprising when considering the $\Lambda$ and $\Sigma^{0}$ are different only in the isospin configuration of the $u$ - $d$ quarks. (The 8effect on kinematics, but kinematics can not explain the differences seen in Fig. 1.) We note that constituent quark models [3] predict a larger coupling constant for t-channel processes for the $\Lambda$ than for the $\Sigma$, which is consistent with the data in Fig. 1.

The unpolarized and interference cross sections for the Lambda (circles) and $\Sigma$ (squares) production are shown in Fig. 2 as a function of $\cos \theta^{*}$. Also shown in this figure are curves from a hydrodynamic model by Mart and Bennhold [4] where the effective degrees of freedom are baryons and baryon resonances (s-channel) and mesons (t-channel), for the $\Lambda$ and $\Sigma$ channels. Although the calculations predict the general magnitude of the unpolarized cross sections $\left(\sigma_{U}=\sigma_{\mathrm{T}}\right.$ $+\varepsilon \sigma_{\mathrm{L}}$ where $T$ and $L$ represent the transverse and longitudinal photon polarizations and 8 is the standard kinematic variable), the interference cross sections for $\Lambda$ production show structure not present in the solid curves. For $\Sigma$ production, the interference structure functions are small, but again differ significantly from the preductions shown in the dashed curves. The calculations are based on phenomenological fits to previous data [5? ] which had much larger error bars. Clearly, the new precise data from CLAS will force improvements in the theoretical models which in turn could lead to a more fundamental understanding of the production mechanism of strange quarks.

Both $K$ and $K^{*}$ mesons, with masses of 494 and $892 \mathrm{MeV}$ respectively, contribute to the t-channel in the $\mathrm{H}\left(e, e^{\prime} K^{+)}\right.$reaction. As a result, any model of this reaction will necessarily predict the coupling constants for $K^{*}$ production. By measuring the $\mathrm{H}\left(e, e^{\prime} K^{*}\right)$ reaction in addition to $K^{+}$ production, tigter constraints may be placed on theoretical models. Production of the neutral $K^{*}$ has significant experimental advantages, because it decays almost immediately into $K+$ and $\pi$ The large acceptance of CLAS provides the kinematic phase space necessary to measure $K^{0} *$ production. Also, t-channel contributions are minimized due to the neutral charge, resulting in the prediction that s-channel nucleon resonances are the primary contributions. This reaction has a threshold just above $W=2.0 \mathrm{GeV}$, and so ite may be sensitive to higher-energy $N^{*}$ resonances. Known $N^{*}$ resonances above $2 \mathrm{GeV}$ are the $\mathrm{G}_{17}(2190), H_{19}(2220)$ and $\mathrm{G}_{19}(2250)$. The $W$ distribution of strength for $K^{0 *}$ production are shown in Fig. 3. We note that the acceptance

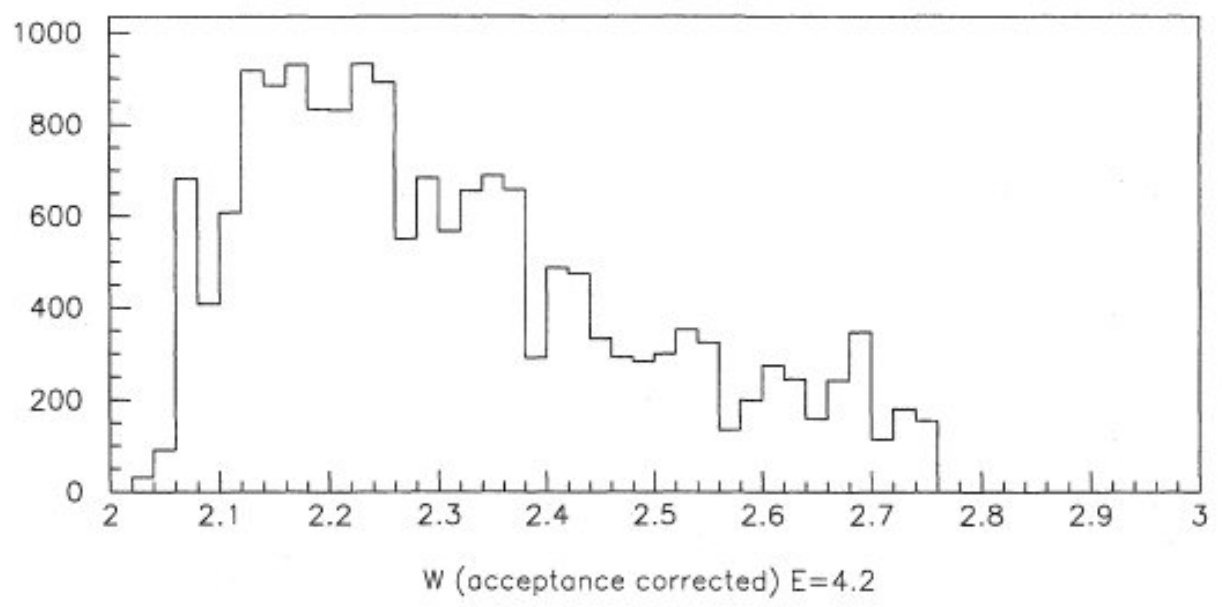

FIGURE 3. Relative yield (vertcal axis) of $K^{*}$ electroproduction as a function of $W$, after correction for the detector acceptance. The threshold for the $\mathrm{H}\left(e, e^{\prime} K^{0 *}\right) \Sigma^{+}$reaction is $W=2.08 \mathrm{GeV}$. 
correction for the CLAS detector has only a small affect on the shape of the W-dependence. This suggests that the excess strength at $2.1-2.3 \mathrm{GeV}$ is due to $N^{*}$ resonance contributions, although it is not clear if the known (high-spin) resonances are contributing strongly here. Calculations are being developed using the same hadrodynamic model as used for $K^{+}$production, and should provide evidence for the structure seen near threshold for $K^{0 *}$ production.

In conclusion, new precise data on strangeness production from CLAS is challenging theoretical models and will hopefully lead to a new understanding of the role of $N^{*}$ resonances in electroproduction reactions.

\section{ACKNOWLEDGMENTS}

Gabriel Niculescu provided invaluable assistance in the preparation of this paper. The author is grateful for financial support from the National Science Foundation.

\section{REFERENCES}

1. Bennhold, C, "Kaon Photoproduction on the Nucleon", in Strange Quarks in Hadrons, Nuclei and Nuclear Matter, edited by K. H. Hicks, World Scientific, Singapore, 2000, pp. 64-73.

2. Brooks, W., Nucl. Phys. A, 664, 1077c (2000).

3. Capstick, S., and Roberts, W., Phys. Rev. D, 58, 1 (1998).

4. Mart, T., and Bennhold, C., Phys. Rev. C, 61,012201 (1999).

5. Bebek, C., Phys. Rev. D, 15, 3082 (1977). 